\title{
Perspective
}

\section{Aligning graduate competence with occupational standards An analysis of medico legal training and practice in Sri Lanka}

\author{
Edussuriya $\mathrm{DH}^{1^{*}}$, Marambe $\mathrm{KN}^{2}$, Abeyasinghe $\mathrm{N}^{3}$, Jayawickramarajah $\mathrm{PT}^{4}$. \\ ${ }^{I}$ Department of Forensic Medicine, University of Peradeniya \\ ${ }^{2}$ Medical Education Unit, University of Peradeniya, \\ ${ }^{3}$ Department of Forensic Medicine and Toxicology, University of Colombo \\ ${ }^{4}$ Medical Education and Research Unit, Eastern University
}

\begin{abstract}
This paper highlights the necessity to aligning graduate competence with occupational standards in workforce development and discusses the existing gap between the service recipient (ministry of justice), service provider (ministry of health), higher education regulatory bodies (University Grants Commission/Sri Lanka Medical Council), the pre service training institutions (ministry of higher education) and students with special reference to medico legal practice.

It considers the options available as continue training medical officers inadequately in the hope that the number of medico-legal consultants would one day be adequate for the needs of the country, since this as a transient period in the development of the speciality increase the production of specialists resulting in diminishing of expectations from the generalist establish protocols for specialist referrals provide a short internship/training program in medico-legal work to all medical officers immediately after graduation restructure the Forensic medicine teaching program. It proposes a way forward with particular reference to instructional reform while identifying constraints and recommendations for its implementation.
\end{abstract}

Key words: Occupational standards; Medicolegal training; Sri Lanka

Copyright: () 2013 Edussuriya DH. This is an open access article distributed under the Creative Commons Attribution License, which permits unrestricted use, distribution, and reproduction in any medium, provided the original work is properly cited.

*Correspondence: deepthiforensic2000@yahoo.com

How to cite: Edussuriya DH, Marambe KN, Abeyasinghe N, Jayawickramarajah PT.Aligning graduate competence with occupational standards An analysis of medico legal training and practice in Sri Lanka. Anuradhapura Medical Journal 2013;7(1):18-20

DOI: http://dx.doi.org/10.4038/amj.v7i1.6139 


\section{Introduction}

The most recent policy document describing the 10 year development strategy of the government of Sri Lanka identifies the "disparity between expected job performance and training" as a problem relevant to human resources development in the health sector(1)The undergraduate medico-legal training program in Sri Lanka was studied in order to determine whether a disparity exists between performance and training.

\section{From trainee to service recipient}

A discrepancy between requirements of the ministry of health(2) and the guidelines for medico legal programs of universities recommended by the regulatory bodies(3) can clearly be identified. For example, the Subject Benchmark Statement (SBS) prepared by the University Grants Commission (UGC) excludes many important aspects of routine medico legal practice. On the contrary the document containing guidelines and specifications on standards and criteria for accreditation of medical schools prepared by the Sri Lanka Medical Council (SLMC) states competencies which maybe beyond the scope of an undergraduate program (4). However in the categorization of skill levels neither the skills nor the appropriate categories (eg., can perform independently, can perform under supervision, has seen the procedure) have been identified while "medico legal requirements" is broadly mentioned as a single skill that "should be performed independently". The discrepancy between the service provider and the pre service training institute is also fairly obvious in the postgraduate arena when analysing the vision and mission statements of the Post Graduate Institute of Medicine where the training of medico-legal specialists is not address adequately(5).

Conversely, the Ministry of Health too is dependent to a large extent on the contribution of professional bodies (eg., boards of study) in deciding and updating standards in human resources for health, at different levels in the health system. However, lack of definite guidelines for roles and functions of specialties within the service proposed by the relevant authorities, has resulted in the Ministry of Health taking a passive role(6) .

Furthermore, another reason for the disparity between requirements of the service provider and the training may be the fact that curricula differ between what is on paper and what is in action specially due to lack of consensus on the medico-legal functions of a medical officer, opportunistic learning, large groups of students, limited staff in the departments of Forensic Medicine in universities and limited/static resources.

\section{Need for action}

The time may come when there would be an adequate number of specialists in Forensic medicine to conduct at least all complex medico-legal functions, with merely the basic functions being performed by the generalists. This could result in Forensic medicine becoming a postgraduate subject with minimal emphasis during the undergraduate program. However the cadre projections of judicial medical officers in the ministry of health show that this is not a situation which would be achievable in the near future. Even though formulation of a mandatory, structured training for those assigned to conduct medico-legal work is evidence for the existence of a service gap, it is necessary to consider the approach to undergraduate medico legal curricula in this context.

\section{The way forward}

If the expectations of the system from medical officers remain unchanged and based on the fairly broad "occupational standards" in medico-legal practice expressed by the health ministry circulars it will be necessary to incorporate a comprehensive list of competencies into undergraduate curricular with methods to ensure its acquisition $(7,8)$. However the changing face of medico legal practice in the country (evidenced by in service training programs for medical officers medico legal, increasing number of specialists) which has resulted in 'clouding' of the role of the medical officer in medicolegal practice has lead to queries in the reality of having such expectations from medical officers.

It is proposed that medico-legal curricular could be structured either in a comprehensive or spiral manner.

\section{A comprehensive undergraduate program}

In the context of constraints to developing and implementing a comprehensive program the following maybe considered. It could be taught as

- A more comprehensive didactic curriculum with a related clinical service.

- Part of the training in a particular established field eg., Forensic radiology with radiology.

- Part of an emerging discipline eg., identification with molecular biology.

- Single-focus courses eg., traumatology, toxicology.

- Interdisciplinary programs in which law and medical students participate together eg., medical law, human rights.

- Integration into the many components of the existing medical program eg.,traumatology with surgery.

\section{A spiral curriculum}

A spiral curriculum where topics are visited and revisited at time intervals, at different levels of intensity, and often with a different emphasis could be considered eg., for undergraduates skill of autopsy examination could be at the "knowledge" level while for postgraduates it should be at "performance" level(9). Arguments for a spiral curriculum include the fact that a medical course should be designed to produce a "stem" or "pluripotential" doctor with generalist training followed by a programme of specialisation. Though this is the basis for the type of 
curriculum present today in Sri Lanka it is doubtful whether there is a real continuum between undergraduate, internship and postgraduate medico-legal training programs.

It is necessary however to reflect on whether this would address the problem of medico-legal competence of the non-specialist medical officer in the absence of changes to the expectations from a non-specialist medical officer.

\section{Limits to medico-legal curricular reform}

1. Lack of consensus on the medico legal role of medical officers

2. Pressure to conform to the requirements of regulating authorities eg., SLMC, UGC and the Quality Assurance and Accreditation Council

3. Increasing expectations of stakeholders eg., patients, ministry of health/ justice

4. Lack of autonomy of universities to dictate the number and the quality of students

5. The need to balance the demands of stakeholders with the increasing number of students and a static resource allocation.

6. Pressure to prioritise the outcomes due to expansion of knowledge with limited curricular time

7. Lack of staff in departments of Forensic medicine in Universities due to inadequate incentives in the ministry of higher education as compared to the ministry of health

8. Less control by Medical faculties over clinical teaching done by specialists of the health ministry
9. An institutional value system which rewards research accomplishments more than educational effectiveness.

10. Work overload in hospitals where students become passive observers instead of active learners.

11. Implementation of academic programs resting mostly on specialist medical teachers with little or no "formal educational training"; who have spent their lifetime in conventional teacher-centred, subject-based schools.

\section{Recommendations}

1. Define the role of a medical officer in conducting medico-legal work ('job description').

2. Revise and align the SBS prepared by the UGC, guidelines and specifications on standards and criteria for accreditation of medical schools prepared by the SLMC, circulars of the ministry of health and forensic medicine curricula of universities.

3. Develop a curriculum where different medico-legal competencies could be acquired at different levels (undergraduate/postgraduate/continuous medical education programs)

4. Determine the level at which a medical officer may deemed to be "specially skilled" in providing expert evidence to court.

5. Develop curricula with emphasis on;

- Acquisition of technical skills related to medicolegal practice eg., history taking, autopsy

- Innovative instructional methods eg., video presentations and role modelling

\section{References}

1. Vision for a New Sri Lanka: The Ten Year Horizon Development Framework. Mahinda Chintana 2006-2016 [cited 27 November 2012]. Available from:http://www.treasury.gov.lk/EPPRM/npd/pdfdocs/budget2007/MahindaChintanaTenYearDevelopmentPlan.pdf

2. Manual of management of base and provincial hospitals and Manual of management of district hospitals, peripheral units and rural hospitals 1995 .

3. Subject benchmark statement in Medicine. Committee of vice chancellors and directors and university grants commission 2006[cited 27 November 2012]. Available from:http://www.qaacouncil.lk/qa_fw/SB_Reports/SBS_Medicine.pdf

4. Guidelines and specifications on standards and criteria for accreditation of medical schools in sri lanka and courses of study provided by them. Sri Lanka Medical council 2011 [cited 27 November 2012]. Available from:http://www.cmb.ac.lk/pgim/

5. Master plan study for strengthening health system in the democratic socialist republic of Sri Lanka 2003 [cited 6 July 2012 ]. Available from:http://gwweb.jica.go.jp/km/FSubject0201.nsf/03a114c1448e2ca449256f2b003e6f57/96dbd84441f528f249257038002f55 4e/\$FILE/03-145_Vol_IJJICA.pdf

6. Edussuriya D, Marambe KN, Abeyasinghe N, Jayawickramarajah PT. Competencies required by medical officers to provide expert medical evidence to court, as perceived by the judiciary: A study conducted in Sri Lanka. Proceedings of the 8th Asia Pacific Medical Education Conference 2011; 26: 162-65.

7. Edussuriya DH, Marambe KN, Abeyasinghe N, Jayawickramarajah PT. An evidence based approach to curriculum development in forensic medicine. Sri Lanka journal of forensic medicine science and law 2012; 3: 25-26.

8. Davis MH, Harden RM. Planning and implementing an undergraduate medical curriculum: the lessons learned. Medical Teacher 2003; 25: 596-608.

9. Ludmerer KM. The Marketplace and Medical Education. The Ochsner Journal 2001; 3: 3-6. 
Submit your next Manuscript to Anuradhapura Medical Journal 